\title{
Possibility of microwave localization to produce an experimental plasma fireball
}

\author{
By Masashi Kamogawa, ${ }^{*), * *), \dagger}$ Haruo TanaKa,${ }^{*)}$ Hideho Ofuruton, ${ }^{* * *)}$ \\ and Yoshi-Hiko OHTSUkI ${ }^{*)}$ \\ (Communicated by Seiya UyEDA, M. J. A., Dec. 13, 1999)
}

\begin{abstract}
We propose, by numerical calculation, a possibility of microwave localization to produce a plasma fireball in the experiment of $2.45 \mathrm{GHz}$ microwave interference. Calculation shows that in one-dimensional system, a few hundred times of intensification of localized microwave could be obtained. The relationship between the position of a plasma fireball production and the intensity of localized microwave is discussed.
\end{abstract}

Key words: Ball lightning; microwave; plasma; localization; discharge.

Introduction. Ball lightning has attracted the interests of many researchers all over the world. ${ }^{1,2)}$ Some of them collected a lot of eyewitness reports of ball lightning. Most of the duration of observed ball lightning ranges from a few seconds to a few minutes. There are many kinds of ball lightning's motion such as going up, down, left and right, moving against the wind, passing through windows without damaging and so on. Kapitza $^{3)}$ suggested that ball lightning should appear at the antinodes of electromagnetic wave of radio frequency between the earth and thunderclouds. Motivated by the suggestion, Ohtsuki and Ofuruton ${ }^{4)}$ succeeded in producing plasma fireballs of which characteristics were similar to that of natural ball lightning. Kondo et $a l .^{5)}$ and Kamogawa et $a l^{6}{ }^{6}$ also reproduced some eyewitness reports. They used a $2.45 \mathrm{GHz}$ microwave oscillator. The maximum power was $5 \mathrm{~kW}$ and plasma fireballs caused by microwave interference appeared in a cylindrical cavity. According to Kapitza, the highest intensity of electromagnetic field to cause microwave discharge should be produced at the antinode of the $\mathrm{H}_{01}\left(=\mathrm{TE}_{01}\right)$ mode ${ }^{7}$ in the cylindrical cavity. However, it can be shown that microwave discharge can

\footnotetext{
*) Department of Physics, Waseda University, 3-4-1 Okubo, Shinjuku-ku, Tokyo 169-8885, Japan.

**) RIKEN International Frontier Group on Earthquake, c/o Earthquake Prediction Research Center, Tokai University, 3-20-1 Orito, Shimizu, Shizuoka 424-8610, Japan.

***) Tokyo Metropolitan College of Aeronautical Engineering, 8-52-1 Minamisenju, Arakawa-ku, Tokyo 116-0003, Japan.

†) Correspondence to: M. Kamogawa.
}

not take place because the amplitude of microwave at the antinode in the $\mathrm{H}_{01}$ mode is only four times as large as that of the incident microwave and is not enough to make discharge. Moreover, the plasma fireball could be produced only when a hand-made cavity was used and not when a machine-made precisely shaped cavity was used. ${ }^{8)}$ Kondo et $a l .{ }^{8)}$ investigated the microwave mode in the cylindrical cavity using thermal paper which becomes burned black when exposed to high intensity microwave. When the hand-made shape cavity was used, there appeared complicated mode which was completely different from the $\mathrm{H}_{01}$ mode generated in the precisely shaped cavity.

On the other hand, Tanaka and Tanaka ${ }^{9)}$ proposed that ball lightning in nature was caused by electromagnetic wave localization. They calculated the intensity of localized electromagnetic field in quasi one-dimensional system, and showed that very high intensity could be obtained.

In this work, we have tried to confirm numerically the possibility of realizing the localized microwave in our $2.45 \mathrm{GHz}$ microwave one-dimensional experiment. Here we consider two types of one-dimensional disordered system such as a quasi-periodic system and a fractal system. Fibonacci chain and Cantor bar ${ }^{10), 11}$ are chosen as a quasi-periodic system and a fractal system, respectively.

Experiments. In order to obtain some experimental parameters, we tried a fundamental experiment in one-dimensional system. A schematic diagram of the experiment is shown in Fig. 1. We used a $2.45 \mathrm{GHz}$ microwave oscillator (magnetron). The microwave 


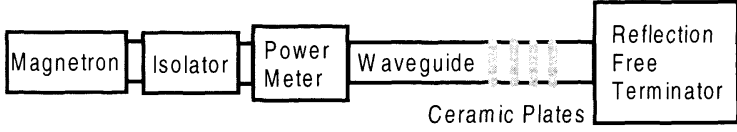

Fig. 1. Schematic diagram of the experiment.

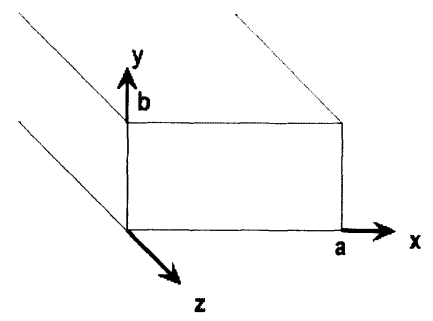

Fig. 2. Coordinates set up in the waveguide.

radiation was guided through a rectangular waveguide. The cross-section was $109 \mathrm{~mm}(=a) \times 55 \mathrm{~mm}(=b)$. The power of incident microwave was $2 \mathrm{~kW}$ and our power meter was able to measure the forward and the reflective powers separately. Isolator set up between the magnetron and the power meter gave the magnetron less damage. Ceramic plates (2.25 mm thick), which were the same size as the cross-section of the waveguide, were put in the waveguide. The direction of the propagation of the microwave was perpendicular to the ceramic plates. A reflection-free terminator was connected at the end of the waveguide.

Model. Here we make a model that reproduces the experimental condition. The coordinates are shown in Fig. 2. Maxwell equations in dielectric medium and the air are given by

$$
\begin{aligned}
& d i v \mathbf{E}=0 \\
& d i v \mathbf{B}=0 \\
& \operatorname{rot} \mathbf{E}=-\frac{1}{c} \frac{\partial \mathbf{B}}{\partial t} \\
& \operatorname{rot} \mathbf{H}=\frac{1}{c} \frac{\partial \mathbf{D}}{\partial t}
\end{aligned}
$$

where $c$ is the light velocity. The constitutive relations are given by

$$
\begin{aligned}
& \mathbf{D}=\varepsilon \mathbf{E} \\
& \mathbf{B}=\mu \mathbf{H}
\end{aligned}
$$

where the dielectric constant $\varepsilon$ is given by

$$
\begin{aligned}
\varepsilon & =1 & & (\text { in the air }) \\
& =\text { constant }(\neq 1) & & (\text { in the dielectric medium }),
\end{aligned}
$$

and the magnetic permeability in the air and the dielec-

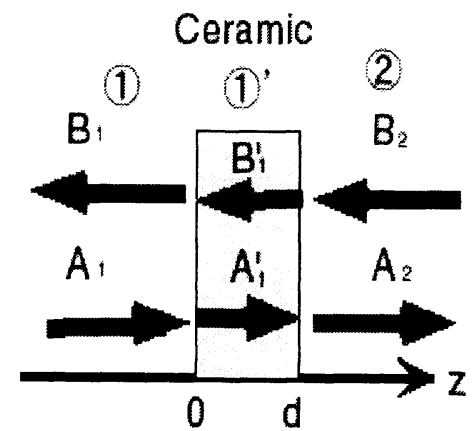

Fig. 3. A ceramic plate is set up from origin to $d$ on $z$-direction. $A_{1}$, $B_{1}, A_{1}^{\prime}, B_{1}^{\prime}, A_{2}$ and, $B_{2}$ are amplitudes of electric field in $+z$ and $-z$ direction in each region, respectively.

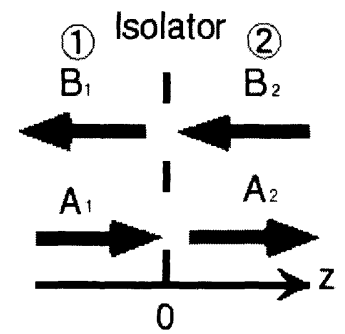

Fig. 4. An isolator behaves like a ceramic plate without thickness. Here, it is set up at origin in $z$-direction. $A_{1}, B_{1}, A_{2}$, and $B_{2}$ are amplitudes of electric field in $+z$ and $-z$ direction in each region, respectively.

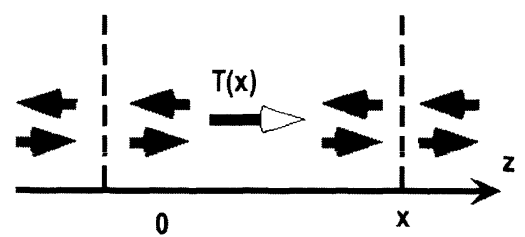

Fig. 5. Translation matrix is defined based on this figure.

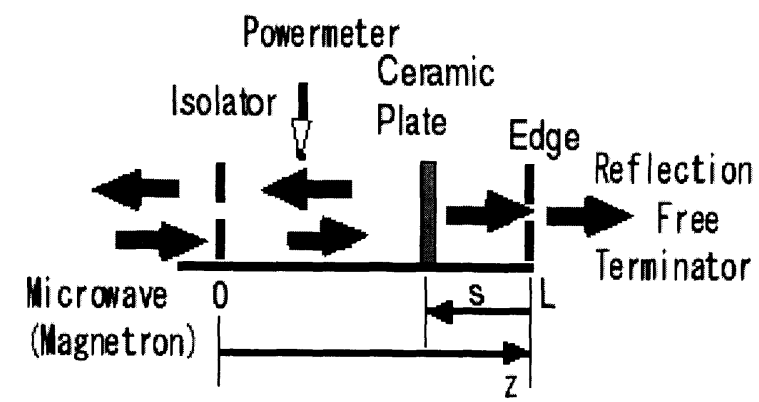

Fig. 6. One dimensional representation of the system depicted iñ Fig. 1.

tric media both is approximately

$$
\mu=1
$$

By considering the $\mathrm{H}_{01}$ mode solution $E(x, z, t)$ on coordinates shown in Fig. 2, Maxwell equations were shown 
Table I. The three parameters obtained by comparison of experiment of data with numerical model

\begin{tabular}{cccc}
\hline & One plate & Two plates & Three plates \\
\hline $\boldsymbol{\varepsilon}$ & 7.0 & 8.0 & 8.0 \\
$\xi$ & 0.04238 & 0.04238 & 0.04238 \\
$L$ & 210.3 & 210.3 & 210.3
\end{tabular}

to become

$$
\begin{aligned}
\frac{\partial^{2} E}{\partial x^{2}}+\frac{\partial^{2} E}{\partial z^{2}} & =\frac{1}{c^{2}} \frac{\partial^{2} E(x, z, t)}{\partial t^{2}} \\
& =-\varepsilon \frac{\omega^{2}}{c^{2}} E(x, z)=-\varepsilon k^{2} E(x, z)
\end{aligned}
$$

where $k$ is the wave number in the air, $\omega$ is the angular frequency of microwave and the $E(x, z, t)$ was given by

$$
E(x, z, t)=E(x, z) \cdot e^{-i \omega t} .
$$

From the boundary condition in $x$-component such as $E(0, z)=E(a, z)=0$, we finally obtained

$$
E(x, z)=\left(A e^{i \kappa z}+B e^{-i \kappa z}\right) \cdot \sin \left(\frac{\pi}{a} x\right)
$$

where the wave number $\kappa$ in the waveguide is given as $\kappa=\sqrt{\varepsilon k^{2}-k_{c}^{2}}$ with $k_{c}=\pi / a$. Also, the wavelength $\lambda_{g}$ in the waveguide was derived by

$$
\lambda_{g}=\frac{2 \pi}{\kappa}=\left\{\varepsilon \lambda^{2}-(2 a)^{-2}\right\}^{-\frac{1}{2}}
$$

where $\lambda$ is the wavelength in the air.

We, then, tried to obtain the transfer matrixes of the ceramic plate and isolator.

(i) Dielectric medium (Ceramic plate) : At first, we consider the electric field in the case of the existence of a dielectric medium ( = a ceramic plate) with finite thickness. The electric field in each region in Fig. 3 is given as

$$
\begin{aligned}
& E_{1}=\left(A_{1} e^{i \kappa z}+B_{1} e^{-i \kappa z}\right) \cdot \sin (\pi x / a) \\
& E_{1}^{\prime}=\left(A_{1}^{\prime} e^{i p z}+B_{1}^{\prime} e^{-i p z}\right) \cdot \sin (\pi x / a) \\
& E_{2}=\left(A_{2} e^{i \kappa z}+B_{2} e^{-i \kappa z}\right) \cdot \sin (\pi x / a) .
\end{aligned}
$$

After the electric field matching at $z=0$ and $z=d$ using (1), (1) and (2), the transfer matrix from region (1) to region (2) was finally obtained as

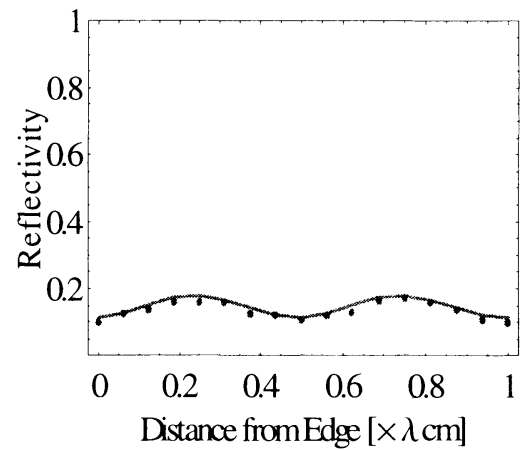

Fig. 7. One plate is set up in the waveguide. Dots mean location of a ceramic plate by the experiment. Solid line is drawn as a numerical result. Horizontal axis is the distance from the edge of the waveguide, which means the value $s$ in Fig. 6 .

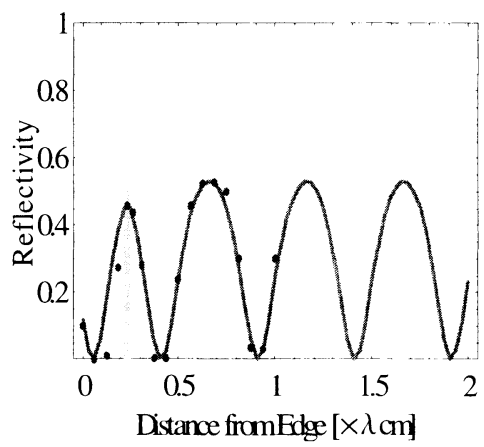

Fig. 8. Two plates are set up in the waveguide. One plate is fixed at $z=0.25 \lambda$. Dots mean location of the second ceramic plate, which is shifted from the edge with the interval $\lambda_{\text {. }} / 16$ except just in front of and behind the fixed plate. Solid line is the numerical result.

$$
\begin{aligned}
\left(\begin{array}{c}
A_{2} \\
B_{2}
\end{array}\right)= & \frac{1}{4 \kappa p}\left(\begin{array}{cc}
i \kappa e^{-1 \kappa d} & e^{-i \kappa l} \\
i \kappa e^{i \kappa d} & -e^{i \kappa d}
\end{array}\right)\left(\begin{array}{cc}
e^{i p d} & e^{-i p d} \\
i p e^{i p d} & -i p e^{-i p d}
\end{array}\right) \\
& \cdot\left(\begin{array}{cc}
-i p & -1 \\
-i p & 1
\end{array}\right)\left(\begin{array}{cc}
1 & 1 \\
i \kappa & -i \kappa
\end{array}\right)\left(\begin{array}{l}
A_{1} \\
B_{1}
\end{array}\right)
\end{aligned}
$$

where the wave number in the air is given by $\kappa=\sqrt{k^{2}-k_{c}^{2}}$ and the wave number in the dielectric medium is given by $p=\sqrt{\varepsilon k^{2}-k_{c}^{2}}$.

(ii) Effective dielectric medium (Isolator): The isolator for giving a magnetron less damage behaves something like a dielectric medium with reflection. The electric field in each region in Fig. 4 is given as

$$
\begin{aligned}
& E_{1}=A_{1} e^{i \kappa z}+B_{1} e^{-i \kappa z} \\
& E_{2}=A_{2} e^{i \kappa z}+B_{2} e^{-i \kappa z}
\end{aligned}
$$

The isolator can be treated as an effective dielectric medium of a $\delta$-function shape with polarizability $\alpha$. From the eqs. [1] [5], substituting $D=E+4 \pi P$,

$$
\frac{\partial^{2} E(x, z)}{\partial x^{2}}+\frac{\partial^{2} E(x, z)}{\partial z^{2}}=-\frac{\omega^{2}}{c^{2}}(1+4 \pi \alpha \delta(z)) E(x, z)
$$


(a)

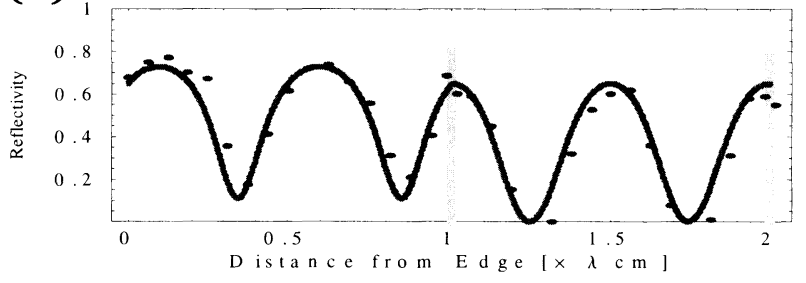

(b)
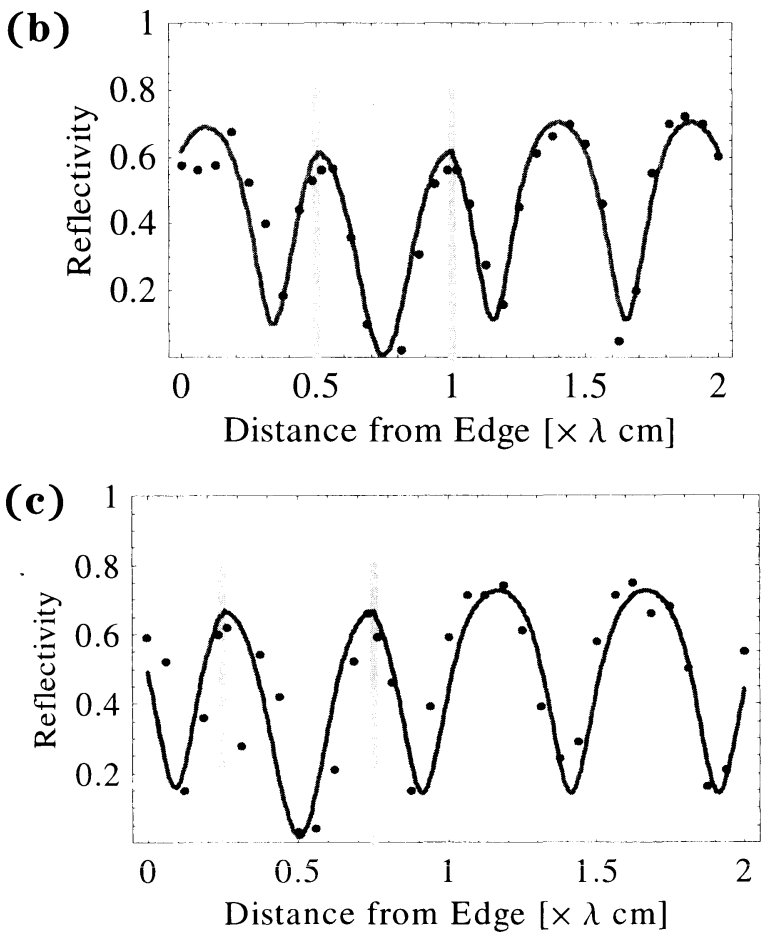

Fig. 9. Three cerarnic plates are set up in the waveguide. Two bars means the locations of the two fixed ceramic plates. Dots mean location of the third ceramic plate, which plate is shifted from the edge with the interval $\lambda_{g} / 16$ except just in front of and behind the fixed plate. Solid line is the numerical result. (a) Two ceramic plates are fixed at $\lambda$, and $2 \lambda$. (b) Two ceramic plates are fixed at $0.5 \lambda$, and $\lambda$. (c) Two ceramic plates are fixed at $0.25 \lambda$, and $0.75 \lambda$.

where the polarization is given by $P=\alpha \delta(z) E(z)$.

From the boundary condition at $z=0$, we obtain

$$
\begin{aligned}
& -E(+0)+E(-0)=0 \\
& -E^{\prime}(+0)+E^{\prime}(-0)=4 \pi \alpha \frac{\omega^{2}}{c^{2}} E(0)
\end{aligned}
$$

From the relations [14], the transfer matrix from region (1) to region (2) is finally obtained as

$$
\left(\begin{array}{l}
A_{2} \\
B_{2}
\end{array}\right)=\frac{1}{2 \kappa i}\left(\begin{array}{cc}
2 \kappa i-\xi & -\xi \\
\xi & 2 \kappa i+\xi
\end{array}\right)\left(\begin{array}{l}
A_{1} \\
B_{1}
\end{array}\right)
$$

where

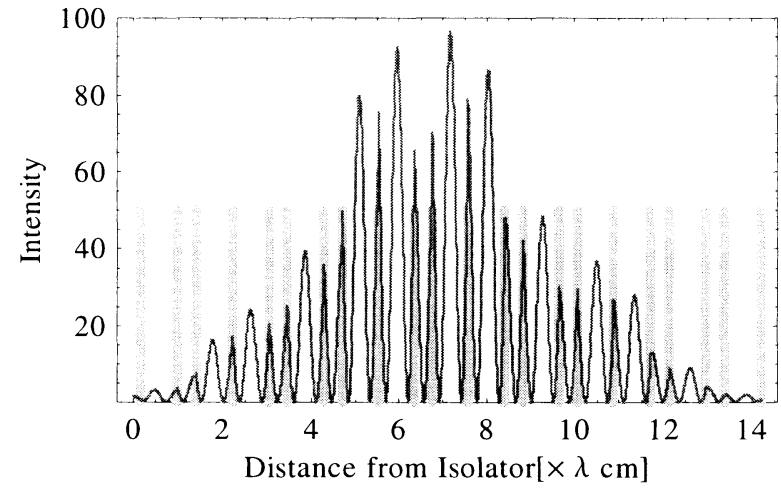

Fig. 10. Results of Fibonacchi Chain arrangement.

$$
\xi=4 \pi \alpha \frac{\omega^{2}}{c^{2}}
$$

To use the transfer matrix [11] and [15] at arbitrary location $x$ (see Fig. 5), we define the translation matrix like

$$
\mathrm{T}(x) \equiv\left(\begin{array}{cc}
e^{i \kappa x} & 0 \\
0 & e^{-i \kappa x}
\end{array}\right) .
$$

Results. The system depicted in Fig. 1 is represented in Fig. 6 as a one-dimensional scheme. To reproduce the experiment numerically, three parameters should be obtained. The parameters are dielectric constant $\varepsilon$ of the ceramic plate in the eq. [4], $\xi$ related to effective dielectric constant in the eq. [16], and length $L$ from the isolator to the end of waveguide in Fig. 6. In the experiment, one plate, two plates, and three plates were set up inside the waveguide. Then, they were shifted step by step with the interval $\lambda_{g} / 16$ to obtain the reflectivity in each position. Dots in Figs. 7-9 are obtained by the experiment. Numerical results are shown by solid lines in Figs. 7-9. By comparing the numerical results and the experimental data, the parameters are obtained, as shown in Table I. Although the dielectric constant $\varepsilon$, unfortunately, have two values, we take $\varepsilon=8.0$ as an assumption.

Then, two kinds of arrangement are taken into account. One is Fibonacchi chain arrangement as a quasi-periodic system and the other is Cantor bar arrangement as a fractal system.

(a) Fibonacchi Chain. Fibonacchi chain of the $m$-th generation is generated by repeating the application of the "inflation" rule. This chain is represented by the sequence of the letter " $A$ " and " $B$ " which stand for two kinds of interval for the ceramic plates, respectively. The " $A B$ " is taken as the first generation. According to the inflation rule, Fibonacchi chain of the next generation is generated by changing the " $A$ " to " $A B$ " and " $B$ " to " $A$ ". 
0

$\mid$

1

$\mid$

2

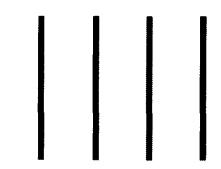

3

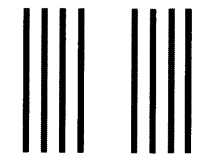

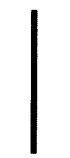
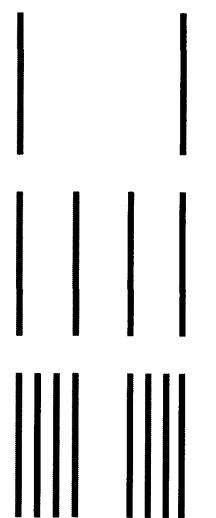

Fig.11. The set of the ceramic plates by Cantor bar.

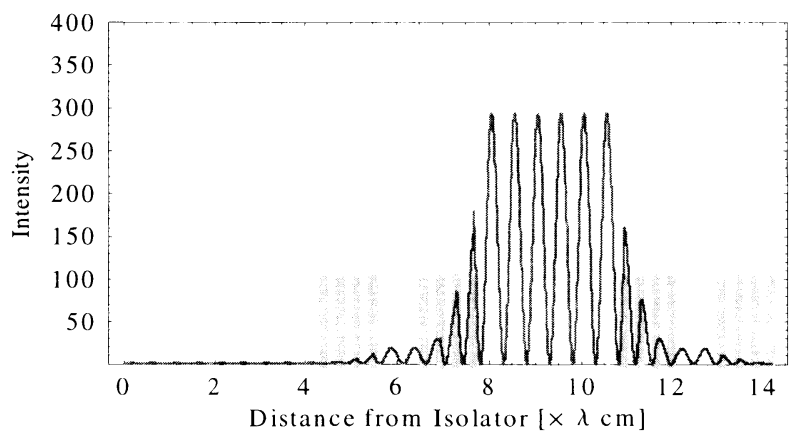

Fig. 12. Results of Cantor bar arrangement.

The higher generation of Fibonacchi chain is obtained by the repeated application of the inflation rule. For example, the sixth generation of Fibonacchi chain is given by

$$
\text { ABAABABAABAABABAABABA. }
$$

In this arrangement [18], 22 ceramic plates should be set up. Substituting some values into " $A$ " and " $B$ " and changing the values, the results of the localization of high intensity of microwave can be obtained. We show one result in Fig. 10. When " $A$ " and " $B$ " are equal to $0.806 \lambda$ and $0.404 \lambda$, respectively, the intensity of the microwave relative to that of the incident wave became approximately 100 times.

(b) Cantor Bar. The arrangement of a Cantor bar is generated by discrete scaling based on a Cantor set and has a fractal structure as shown in Fig. 11. The arrangement of ceramic plates of the zero-th generation of a Cantor bar is made by setting up two ceramic plates with the interval $l$. In the first generation, ceramic plates are added at $l / 3$ and $2 l / 3$ of the zero-th generation. In the (a)

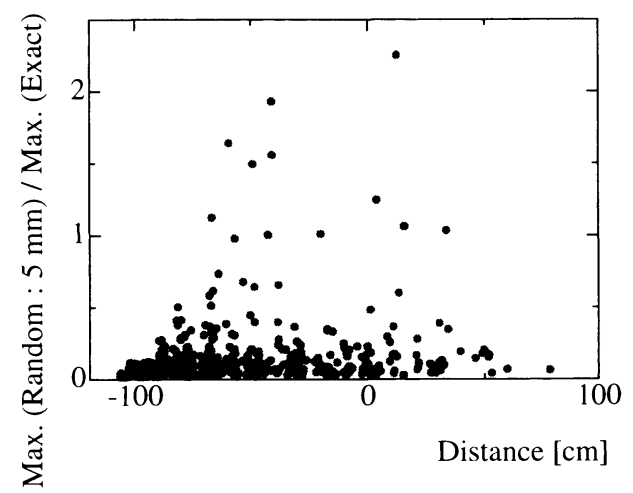

(b)

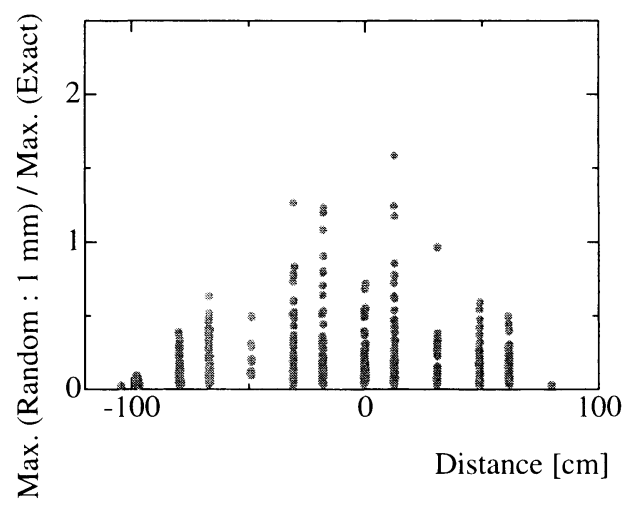

(c)

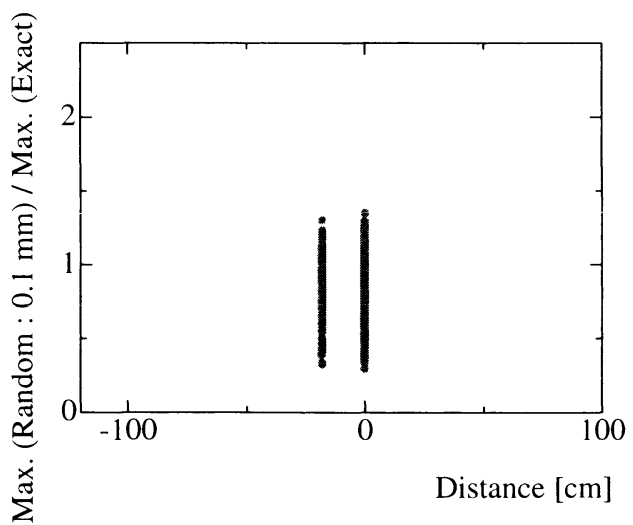

Fig. 13. Numerical experiments based on Fibonacchi-chain depicted in Fig. 10. All ceramic plates were randomly shifted 1000 times from exact position in Fig. 10 within 5 mm, $1 \mathrm{~mm}$, and $0.1 \mathrm{~mm}$. Vertical axis means the maximum intensity of each time divided by that of the case of exact position in Fig. 10. Horizontal axis is the distance of the maximum location difference. (a) Results of $5 \mathrm{~mm}$ case. Most of the rate of the maximums were much less than 1 . (b) Results of $1 \mathrm{~mm}$ case. The percentage of the results more than $90 \%$ rate is about $0.8 \%$.(c) Results of $0.1 \mathrm{~mm}$ case. Most of the rates were nearly 1 and most of the maximum position were located near or in the position in Fig. 10. The percentage of the results more than $90 \%$ maximum intensity in Fig. 10 is more than $60 \%$.

second generation, the arrangement reduced to a scale of one-third of the arrangement of the first generation is embedded into $0 \sim l / 3$ and $2 l / 3 \sim l$. In the same way, 
the third generation is obtained as shown in Fig. 11. The condition in the fourth generation is calculated as shown in Fig 12 . Taking $l=9.79 \times \lambda$, approximately 300 times intensity to relative to the incident intensity can be found.

Results of numerical experiment are shown in Fig. 13 for the case of Fibonacchi chain in Fig. 10. All ceramic plates were randomly shifted 1000 times from the exact position in Fig. 10 within $5 \mathrm{~mm}, 1 \mathrm{~mm}$, and $0.1 \mathrm{~mm}$. In $0.1 \mathrm{~mm}$ case, the numerical experiments showed that $60 \%$ of 1000 trials resulted in over $90 \%$ intensity of the no-shift case in Fig. 10.

Concluding remarks. We calculated the intensity of $2.45 \mathrm{GHz}$ microwave inside a rectangular waveguide for both a quasi-periodic system and a fractal system. In both systems, we found that the intensity of the microwave is considerably enhanced in restricted regions. Sometimes the enhancement of intensity exceeded a hundred times. However, a few hundred times enhancement is not enough to make microwave discharge for localized plasma as a fireball in this experimental condition. In our experiment, when the power of magnetron is $2 \mathrm{~kW}$, the amplitude of the electric field of incident microwave becomes $10^{4} \mathrm{~V} / \mathrm{m}$. Then the highest amplitude of the electric field of the localized microwave should become only $10^{5} \mathrm{~V} / \mathrm{m}$. The minimum electric field required for the discharge is probably about $10^{6} \mathrm{~V} / \mathrm{m}$. Therefore the amplitude of the localized electric field amounts only to one-tenth of that required for the discharge. To realize localized plasma discharge in this experimental condition, a small pointed metallic object like a needle should be set up between the ceramic plates around the highest intensity. It would be effective to make the intensity of electromag- netic field, especially electric field, higher around the needle to cause microwave discharge without disturbing the total electromagnetic field in the waveguide. If a longer waveguide and many more ceramic plates can be used, a localized plasma fireball can probably appear by microwave localization only.

Acknowledgments. The authors would like to thank Dr. S. Uyeda, M. J. A., (RIKEN), and Mr. N. Fujiwara (Waseda University) for their help, useful comments and advice.

\section{References}

1) Singer, S. (1971) The Nature of Ball Lightning. Plenum Press, New York.

2) Barry, J. D. (1980) Ball Lightning and Bead Lightning. Plenum Press, New York.

3) Kapitza, P. L. (1955) Dokl. Akad. Nauk. (in Russian) 101, 245248 (English translation); Kapitza, P. L. (1965) In Collected Papers of Kapitza (ed. D. Ter Haar). vol. 2, Pergamon, New York, pp. 776-780.

4) Ohtsuki, Y.-H., and Ofuruton, H. (1991) Nature 350, 139-141.

5) Kondo, N., Ofuruton, H., and Ohtsuki, Y.-H. (1997) Jour. of Atmos. Electr. 17, 47-51.

6) Kamogawa, M., Ofuruton, H., Kondo, N., and Ohtsuki, Y.-H. (1998) J. of Atmos. Electr. 18, 47-51.

7) Jackson, J. D. (1975) Classical Electrodynamics, 2nd ed., John Wiley \& Sond, New York, pp. 345-346.

8) Kondo, N., Kamogawa, M., Ofuruton, H., and Y.-H., Ohtsuki (1998) J. of Atmos. Electr. 18, 53-57.

9) Tanaka, K., and Tanaka, M. (1997) Appl. Phys. Lett. 71, 37933795.

10) Schroeder, M. (1991) Fractals, Chaos, Power Laws: Minutes from an Infinite Paradise, W. H. Freeman and Company, New York.

11) Cusack, N. E. (1987) The Physics of Structure disordered Matter, Chapter 9, 10, 11, Adam Hilger Published, U. K. 